\title{
Is nerve sparing clitoroplasty really a nerve sparing?
}

\author{
Waleed Dawood* ${ }^{*}$, Dina Abdallah, Ashraf Soliman, Doaa Khater, Shaymaa Elsayed, Magdy Omar \\ and Haytham Badawy
}

\begin{abstract}
Background: Surgery for clitoral reduction has been practised for over half a century. The aim of clitoral surgery is to achieve normal clitoral morphology without compromising sexual function. To achieve these purposes, many techniques were developed since Young first reported reduction clitoroplasty in 1937. As the importance of the neurovascular bundle was realized, attempts were made to preserve this structure aiming at preservation of the sensory supply to the clitoris to achieve sexual satisfaction.
\end{abstract}

Methods: The study was conducted on six patients with congenital adrenal hyperplasia, who were operated upon with reduction clitoroplasty with neurovascular bundle preservation as a part of the genital reconstruction; surgery was done by single author (first author). Whole surgically removed corporal bodies were processed. Examination of the sections was done for the presence of nerve bundles related to the anatomical location of the neurovascular bundle using $\mathrm{S} 100$ immunohistochemical staining.

Results: In four cases, the presence of nerve fibers in the removed part of the corpus stained by S100 was high on the dorsal surface, with the presence of large nerve fibers (score 3-4), and low on the ventral surface with the presence of small nerve fibers (score 1). In the other two cases, it was low on both surfaces (score 1).

Conclusion: Subfacial clitoroplasty technique carries an inevitable risk of nerve injury and should be replaced by the subtunical technique.

Level of evidence: IV. Type of study: case series with no comparison group.

Keywords: Neurovascular sparing clitoroplasty, Reduction clitoroplasty

\section{Background}

Surgery for clitoral reduction had several milestones in its evolution. The aim of clitoral surgery is to achieve normal clitoral morphology without compromising sexual function [1]. Young first reported reduction clitoroplasty in 1937, the technique of reduction involved reducing the corporal longitudinal dimension, but the glans suffered from ischemia and vanished later. Hence, the technique of clitorectomy was introduced supported by the theory that the clitoris had no function [2].

\footnotetext{
*Correspondence: drwaleeddawood@yahoo.com
}

University of Alexandria, Alexandria, Egypt
Patients who had clitorectomy were shown to have sexual problems; hence, attempts were restarted to perfect a technique of clitoral reduction with preservation of the glans clitoris [3]. Lattimer in 1961 described a technique that preserves glans sensitivity with repositioning of the glans in a normal position [4]. Attempts were made to preserve this important neural and vascular network. Some of these techniques involved dissection of the neurovascular bundles off the corpora, but this exposed them to possible damage. Hence, in 1982 Rajfer et al. [5] suggested a ventral approach to prevent damage to the neurovascular bundle.

In 1983, Kogan et al. [6] suggested a technique of subtunical reduction, which allowed adequate reduction with no damage to the neurovascular bundles. The functional 
outcome of all techniques is not perfect. Surgery was also associated with sexual difficulties [7, 8]. Clitoral surgery is definitely affecting the sensation and orgasm to some extent which should be minimized by proper choice of surgical technique and refinement of procedures.

In this study, we are evaluating the presence of major nerve elements at the anatomical location of the neurovascular bundle over the excised clitoral bodies after ventral nerve sparing clitoroplasty as an indication of possible sensory loss and future sexual dissatisfaction in spite of the proper dissection and preservation of the proper surgical plane between the neurovascular structure and the corporal body.

\section{Methods}

The study was conducted on six patients with congenital adrenal hyperplasia, who were operated upon with reduction clitoroplasty with neurovascular bundle preservation as a part of the genital reconstruction. Children presenting to our institution endocrinology and pediatric urology units with CAH not previously repaired are chosen and examined consecutively. The study was submitted to and approved by our institution ethics committee.

All cases were surgically performed by a single pediatric urologist (first author). The pathology and immunohistochemistry were performed by the single pathologist in our study who processed sectioned stained and photographed the specimen and slides. Surgery utilizing $2.5 \mathrm{X}$ magnifying optical loops was done in all cases for proper dissection and preservation of the neurovascular bundle.

In all cases, ventral nerve sparing clitoroplasty was performed using the following steps. We start our repair by identification of the urogenital sinus and mobilization of the urogenital sinus either partially or totally according to the confluence of the urogenital sinus, and then, complete degloving of the clitoris using a circumcising incision and a plane similar to the plane of hypospadias between buck's fascia and the dartos follows.

We start dissection of the neurovascular bundle from the 5'o clock position of the corpus under the bucks fascia but over the tunica albuginea lifting off the bundle till the route of the corpus clitoris, and then, we control the route by a transfixation suture using $4 / 0$ polyglactin suture; then, complete excision of the corpus is performed after marking the dorsal surface and tip of the corpus by a $5 / 0$ prolene suture, and then, we fix the glans to the underlying route of the corpus using $5 / 0$ polyglactin suture (Figs. 1, 2). We did not do reduction glanuloplasty in any of the children. All children were Prader 3 and more.

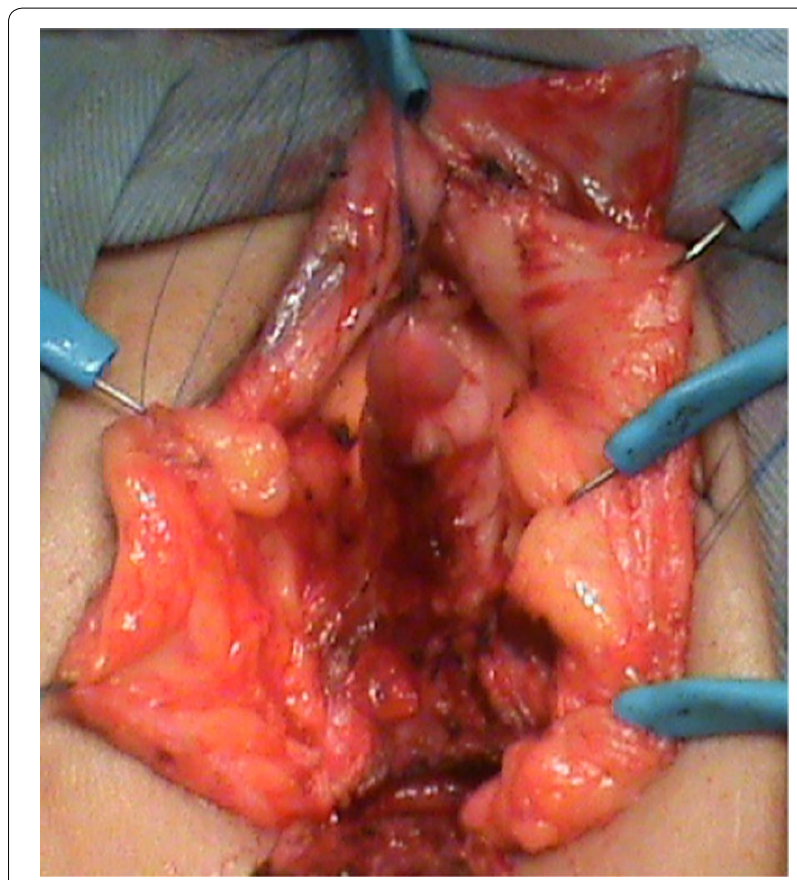

Fig. 1 The clitoris is fully degloved till the penopubic junction

Whole surgically removed clitoris was processed, fixed in $10 \%$ formaldehyde and routinely processed for paraffin embedding. Histological sections $(4 \mu \mathrm{m}$ thick) were stained with hematoxylin and eosin. Immunohistological staining using S100 stain followed.

For immunohistochemical analysis, we used S100 (mouse monoclonal antibody, dilution 1:100). The expression of S100 by peripheral glial cells seems to be a distinctive fact of these cells, whatever their localization and either myelinated or not. S100 proteins are expressed in satellite cells of sensory, sympathetic and enteric ganglia and also in supporting cells of the adrenal medulla Schwann cells in the nerve trunks and the Schwann-related cells of sensory corpuscles. Also, S100 proteins are expressed in peripheral neurons [9].

Immunostaining was performed using streptavidinperoxidase complex method. The immunoreactivity of S100 was evaluated according to the intensity and percentage of positively stained cells. The percentage of positively stained cells was graded as follows: grade $0,0-39 \%$; grade 1, 40-69\%; grade 2,>70\%. Immunostaining intensity was rated as follows: 0 , negative; 1 , weak; and 2, strong. In addition, an Immunoreactive score was calculated by the addition of the percentage score of positively stained cells and the score of staining intensity $(0-4)$. Specimens with an Immunoreactive score of $0-1$ were designated 'low', and those with an Immunoreactive score of 2-4 were designated 'high. 


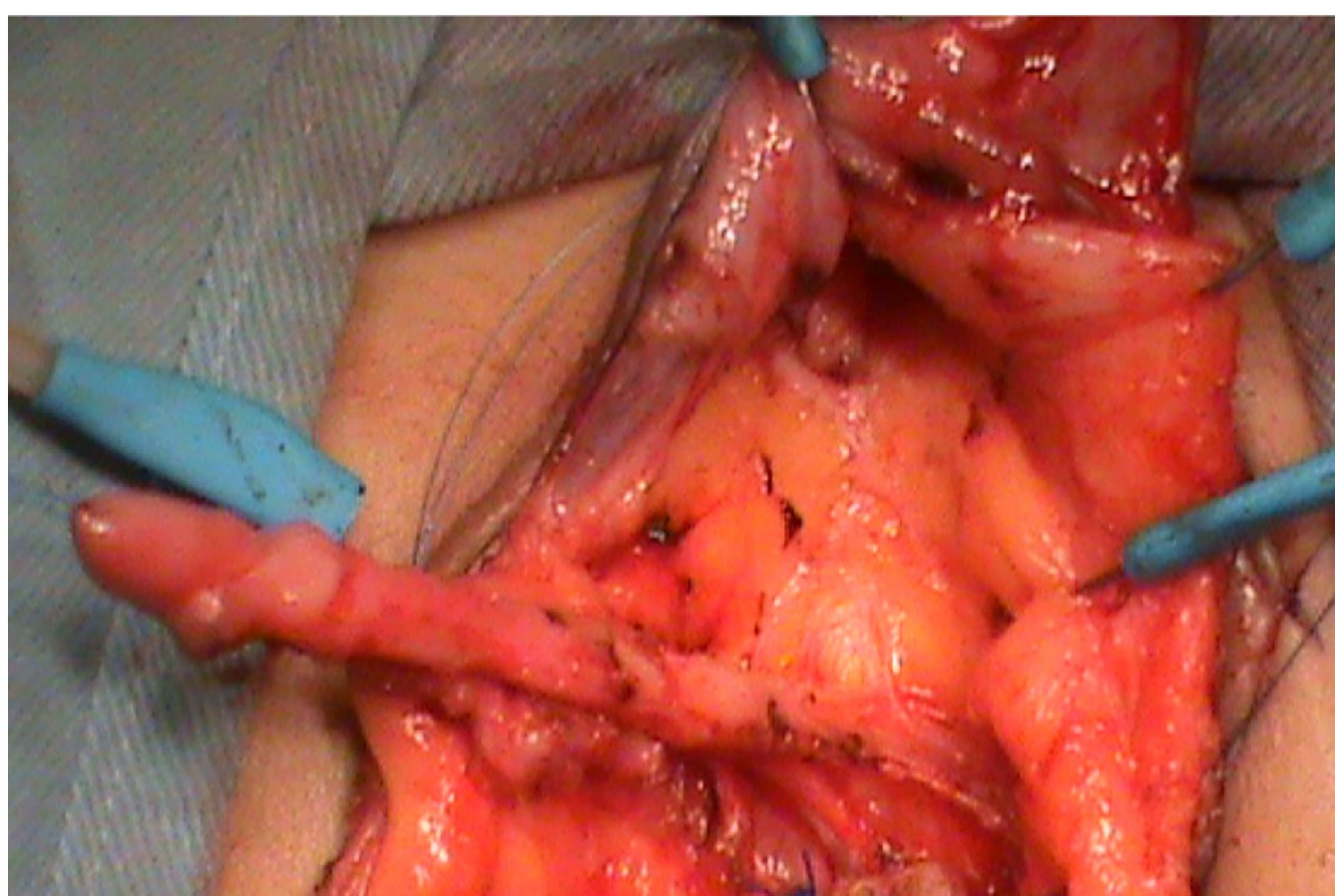

Fig. 2 Full dissection of the neurovascular bundle of the glans clitoris and excision of the body of clitoris after control of the crura of body clitoris

\section{Results}

Six children with congenital adrenal hyperplasia underwent single stage clitorovaginoplasty in whom the corpus clitoris was excised with the tunica albuginea intact and then stained with hematoxylin and eosin, and then, immunohistochemical staining using S100 was performed in all specimens.

The structure of the surgically removed clitoris was comparable to normal clitoris histology being composed of two separate corporal bodies with a septum in between and enveloped in the tunica albuginea stratum.

In four cases, S100 expression was highly intense on the dorsal surface, with the presence of large nerve fibers (score 3-4) and with low intensity on the ventral surface with the presence of small nerve fibers (score 1). In the other two cases, it was low on both surfaces (score 1) (Figs. 3, 4, 5). This means that nerve injury in this clitoroplasty technique is inevitable.

\section{Discussion}

The aim of clitoral surgery is to achieve normal clitoral morphology without compromising sexual function. In this study, all patients were operated upon by a single experienced surgeon considering all the precautions to avoid neurovascular bundle damage and grossly the neurovascular bundle was preserved in all patients by

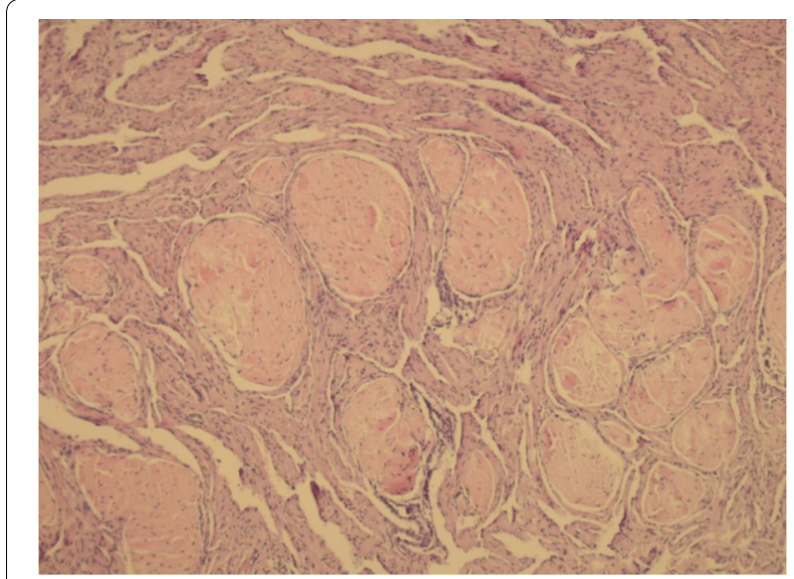

Fig. 3 H\&E stained slide showing thick nerve bundles on the dorsal surface of one case $(\times 100)$

careful dissection in the plane, utilizing fine instruments, using magnifying surgical loops.

We used the ventral approach suggested by Rajfer et al. to prevent damage to the neurovascular bundle in all cases in which we started dissection ventrally at 5'o clock position in the plane between the neurovascular bundle superiorly and the intact tunica albuginea inferiorly till the 7'o clock position on the other side encircling the corpus and lifting up the neurovascular 


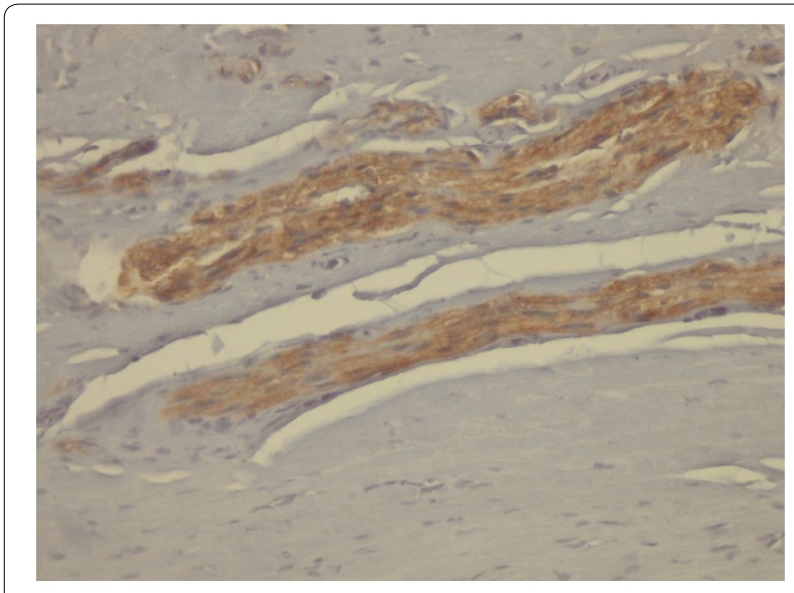

Fig. 4 High S100 immunoreactivity in large nerve bundles on the dorsal surface $(\times 400)$

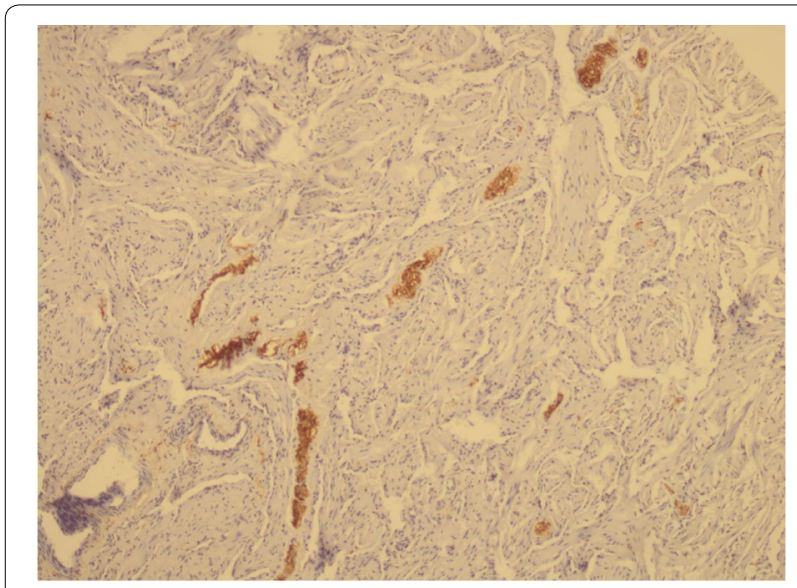

Fig. 5 S100 immunoreactivity in small nerve bundles on the dorsal surface $(\times 400)$

bundle [5]. Still, in four of six cases (66\%) thick nerve fibers can be seen on the dorsal aspect of the clitoral bodies at the anatomical location of the neurovascular bundle imbedded in the fibrous tissue of the clitoris.

Being embedded in the fibrous tissue raises the concern that many penetrating nerves are present even inside the corpus under the tunica envelope which makes the preservation of the neurovascular bundle impossible without resecting a part of the corporal bodies with it as described by Kogan et al. [6] in the technique of subtunical reduction. The long-term evaluation of operated cases of CAH supports the possibility of inevitable neural damage with variable degrees of functional disabilities.

In another study, Melbourne group in 2007 utilizes the subtunical dissection with preservation of the neurovascular bundle through the last 30 years of practising feminizing genitoplasty in children with good outcome due to proper preservation of the nerve fibers of the glans [1].

Crouch et al. presented their experience after feminizing genitoplasty in London. They concluded that sensitivity was decreased in the genital areas where feminizing genitoplasty had been done. Surgery was also associated with sexual difficulties [7].

Creighton published her article under the title 'London experience' and concluded that there is very scanty evidence of a satisfactory post-pubertal cosmetic or anatomical outcome and it is unacceptable to claim that clitoral surgery does not affect sexual function, although the magnitude of this effect needs further evaluation [10].

Rieder and Hurwitz in their evaluation of the results of childhood feminizing surgery could have a complete evaluation in seven patients. Three patients (43\%) had strong, three $(43 \%)$ had moderate, and one (14\%) had no sexual pleasure with clitoral stimulation. Three (43\%) had strong, two (28\%) had mild, and two (28\%) had no orgasms with clitoral stimulation [8].

Lesma et al. evaluated the thermal and vibratory sensitivity of the clitoris, together with the psychosexual outcome of CAH patients operated upon with PasseriniGlazel feminizing genitoplasty. Their inclusion criteria were adult age and penetrative vaginal intercourse. A total of 12 patients (10\% of their operated CAH cases) entered the study. Thermal and vibratory clitoral sensitivity was significantly decreased in all patients compared to healthy controls [11]. Poppas et al. examined the erectile tissue removed from 27 female patient with $\mathrm{CAH}$ for the presence of nerves. In their methodology, they counted the nerves outside the tunica albuginea. In four of 27 patients (15\%), no dorsal nerve branches were visualized in excised erectile tissue; in another 18 patients (67\%), ten or fewer nerve branches were found. In patients who underwent nerve sparing ventral clitoroplasty, 92\% of dorsal nerves detected were $90 \mu \mathrm{m}$ or less. In their study, they counted the nerves outside the tunica albuginea; still, only four cases (15\%) show no dorsal nerve branches in the excised specimen [12].

In our study, thick nerve fibers are encountered on the dorsum of the clitoris corporal body in four specimens and thin nerve fibers in the remaining two specimens. This means that even with careful dissection of the neurovascular bundle outside the tunica albuginea under the buck's fascia, still there are nerves being injured and severed with the transected corpus clitoris.

These are peripheral nerves, whether they regrow again or not is unknown, the effect of cutting such nerves on the clitoral sensitivity is unknown so, and surgeon has to try to preserve such nerves to the maximum and utilize 
the technique that is more trustable in preserving such nerves. Whether the technique of ventral evacuation of the clitoris from erectile tissue is the best for preserving neurovascular bundle or not, we still have no answer for this question, but we are going to shift our surgical clitoroplasty from the lifting of neurovascular bundle above the tunica albuginea to start doing the ventral approach of just removing the erectile tissue leaving intact the tunica albuginea with all nerves outside the tunica intact.

One of the limitations of our study is that we used S100 stain which is a nonspecific stain by which we cannot distinguish between subtypes of nerve bundles, but we expected to deal only with sensory fibers. And also, we did not count the nerve fibers present in the specimens subjected to the study.

Our study is characterized by the presence of unified technique practised by a single surgeon for long time even before this study, pathology and staining were done by one expert pathologist and all children had congenital adrenal hyperplasia; however, the number of children is small and we need also to examine prospectively all specimens taken by subtunical technique of evacuation of erectile tissue to be more sure that nerves are being truly preserved on utilizing this procedure. This type of surgery needs special experience and a good team work which is achieved well in our work.

\section{Conclusion}

Inevitable nerve damage occurs with the subfacial nerve sparing clitoroplasty procedure even with considering all the technical precautions. So, we recommended the subtunical reduction clitoroplasty which is a technique to preserve the intratunical nerves to avoid sexual dissatisfaction in the future. Further evaluation of this topic with larger number of patients is recommended to obtain stronger evidence.

\section{Acknowledgements}

None.

\section{Authors' contributions}

WD had the role of writing, doing surgeries and coordinating between investigators. DA examined the histopathology of specimens, and AS gave the idea of research and an assistance in surgeries and statistics. DK gave the medical support for cases and wrote the discussion, and SE also gave medical support of cases and wrote the background. MO supervised and revised the medical work and the manuscript. HB supervised and analyzed the results and statistics. All authors read and approved the final manuscript.

\section{Availability of data and materials}

The datasets used and/or analyzed during the current study are available from the corresponding author on reasonable request.

\section{Ethics approval and consent to participate}

The study was submitted to and approved by our ethics committee of Faculty of Medicine, Alexandria University. Ethics committee's reference number was not available. Formal written consent was taken from all participants. Consent for publication was taken from all participants.

\section{Consent for publication \\ Done and approved.}

\section{Competing interests}

The authors declare that they have no competing interests.

Received: 19 December 2019 Accepted: 24 June 2020

Published online: 07 September 2020

\section{References}

1. Lean W, Hutson J, Deshpande A, Grover S (2007) Clitoroplasty: past, present and future. Pediatr Surg Int 23:289-293

2. Young H (1992) Genital abnormalities, hermaphroditism and related adrenal disease. Williams and Wilkins, Baltimore (1937); pp 103-105 quoted from Vesely J., Baøinka L., Santi P., Berrino P. and Mugianu M. Reconstruction of the penis in transsexual patients. Acta Chir Plast 34:44-54

3. Mininberg DT (1981) Phalloplasty in congenital adrenal hyperplasia. J Urol 128:355-356

4. Lattimer JK (1961) Relocation and recession of the enlarged clitoris with preservation of the glans: an alternative to amputation. J Urol 86:113-116

5. Rajfer J, Ehrlich R, Goodwin W (1982) Reduction clitoroplasty via ventral approach.J Urol 128:341-343

6. Kogan S, Smey P, Levitt S (1983) Subtunical total reduction clitoroplasty: a safe modification of existing techniques. J Urol 130:746-748

7. Crouch N, Mei Liao L, Woodhouse C, Conway G, Creighton S (2008) Sexual function and genital sensitivity following feminizing genitoplasty for congenital adrenal hyperplasia. J Urol 179:634-638

8. Riedre J, Hurwitz R (2009) Long term anatomical and functional outcomes in patients with disorders of sexual development. J Urol 181(suppl 4):27-31

9. Albuerne M, Mammola CL, Naves FJ, Levanti B, Germanà G, Vega JA (1998) Immunohistochemical localization of S100 proteins in dorsal root, sympathetic and enteric ganglia of several mammalian species, including man. J Peripher Nerv Syst 3(4):243-253

10. Creighton $\mathrm{S}$ (2004) Long-term outcome of feminization surgery: the London experience. BJU Int 93(Supl. 3):44-46

11. Lesma A, Bocciardi A, Corti S, Chiumello G, Rigatti P, Montorsi F (2014) Sexual function in adult life following Passerini-Glazel feminizing genitoplasty in patients with congenital adrenal hyperplasia. J Urol 191:206-211

12. Poppas D, Hochsztein A, Baergen R, Loyd E, Chen J, Felsen D (2007) Nerve sparing ventral clitoroplasty preserves dorsal nerves in congenital adrenal hyperplasia. J Urol 178(4):1802-1806

\section{Publisher's Note}

Springer Nature remains neutral with regard to jurisdictional claims in published maps and institutional affiliations. 